\title{
Quantum properties of light propagating in a coherent-population-oscillation storage medium
}

\author{
P. Neveu, ${ }^{1}$ F. Bretenaker, ${ }^{1,2}$ F. Goldfarb,${ }^{1}$ and E. Brion ${ }^{1,3}$ \\ ${ }^{1}$ Laboratoire Aimé Cotton, Université Paris-Sud, ENS Paris-Saclay, CNRS, Université Paris-Saclay, F-91405 Orsay, France \\ ${ }^{2}$ Light and Matter Physics Group, Raman Research Institute, Bangalore 560080, India \\ ${ }^{3}$ Laboratoire Collisions Agrégats Réactivité, IRSAMC \& UMR5589 du CNRS, Université de Toulouse III Paul Sabatier, \\ F-31062 Toulouse, France
}

(Received 12 February 2019; published 12 July 2019)

\begin{abstract}
We study the propagation and storage of a quantum field using ultranarrow coherent population oscillations (CPOs) in a $\Lambda$-type atomic medium. The predictions for classical fields are checked experimentally in a vapor at room temperature. We derive the evolution of its squeezing spectrum in the presence of a large classical pump field which enables CPOs to exist. We show that the spontaneous emission of the residual population pumped into the excited state progressively destroys the quantum noise properties of the quantum field along propagation. The output quantum field therefore tends to be a coherent state, discarding the possibility to store quantum states of light with CPO.
\end{abstract}

DOI: 10.1103/PhysRevA.100.013820

\section{INTRODUCTION}

One of the prerequisites for implementing quantum information processing is the availability of quantum memories, i.e., quantum devices able to faithfully store quantum states and release them on demand with high fidelity [1]. Since photons appear as natural information carriers, much effort has been devoted to the development of quantum memories for light states during the last 20 years. The most common protocol exploits the strong dispersion which arises together with the electromagnetically induced transparency (EIT) phenomenon: A very narrow transmission resonance can be obtained when two optical transitions couple two ground states to the same excited state in a $\Lambda$ system $[2,3]$. Using this two-photon resonance, light pulses can be stored in the Raman coherence between both lower states of such a $\Lambda$ system in cold atoms [4] or atomic vapors [5,6] using close to or far off optical resonance schemes, as well as in ion Coulomb crystals [7] or rare-earth ions in matrices [8]. EIT-based storage in warm vapors was also demonstrated to preserve single-photon [9] or squeezed [10] states of light. Propagation under such EIT conditions can be described as the interplay between a light field and the Raman coherence, embodied by the dark-state polariton [11]. One drawback of this storage protocol is the very high sensitivity of the coherence, and thus of the dark-state polariton, to dephasing effects induced by the environment, which quickly destroy the memory. This motivated the development of long-lived memories exploiting, for example, Zeeman spin orientation coherences $(\Delta m=1)$ in a gas cell, more robust than the $\Delta m=2$ coherences usually used for EIT-based memories [12] or optimizing dynamical decoupling schemes in rareearth doped crystals [13,14]. Other protocols were proposed which use populations to extend the lifetime of photon-echobased quantum memories $[15,16]$. The quantum properties of such storage schemes are nevertheless controversial because of the semiclassical nature of the atomic populations.
Coherent population oscillations (CPOs) have been shown to offer another way to efficiently store classical light pulses in a $\Lambda$ system [17-19]. Their phase preservation properties even allowed one to store and retrieve the orbital angular momentum of light [20]. The physics of the CPO phenomenon is very different from EIT. It was first identified in a two-level system excited close to optical resonance by two coherent light fields, a strong one called the pump and a weaker one called the probe [21]. When these fields are slightly detuned from each other, intensity beats are induced. If this intensity modulation is slow enough, i.e., if the beat frequency is smaller than the excited-state decay rate, atomic populations then experience a dynamical saturation and adiabatically follow the intensity variations. This population difference modulation leads to an amplification of the light modulation depth. This effect can be seen as a transmission window being opened for the probe beam within the absorption linewidth of the transition. $\mathrm{CPO}$ resonances have been observed in various systems such as bulk defects [22,23], quantum dots [24], fibers [25], and vapors [20], with linewidths limited by the upper-level decay rate: They are thus usually larger than EIT resonances between ground or metastable states, which do not experience spontaneous emission. However, the CPO resonance can become dramatically narrow in a $\Lambda$ system, when two antiphase $\mathrm{CPO}$ phenomena occur along the two legs and combine in an effective so-called ultranarrow CPO between the two longlived ground states of the system [26]. As in the EIT case, it is possible to model the propagation under such $\mathrm{CPO}$ conditions as the interplay between a light field and the ground-state population imbalance [27] embodied by the so-called populariton. As populations are not sensitive to dephasing effects contrary to Raman coherences, the CPO-based storage of classical light fields was demonstrated to be quite immune to perturbations such as magnetic field inhomogeneities [18]. Nevertheless, the question whether quantum properties can or cannot be preserved in a CPO-based storage protocol has not yet been addressed. The aim of the present paper is thus to investigate 
the noise properties of a quantum light field propagating under such ultranarrow $\mathrm{CPO}$ conditions.

To answer this question, Sec. II presents the $\Lambda$ system and the excitation scheme, together with the experimental results obtained in metastable helium for a weak classical probe field. These observations are in excellent agreement with the theory published in Ref. [27]. Section III extends the previous semiclassical theoretical treatment to quantized states of probe field light, while the driving field remains classical. We then derive the evolution of the probe field quantum noise along propagation, with methods similar to the ones used in Ref. [28]. This approach is then applied in Sec. IV to a firstorder derivation of the modifications of the field fluctuations due to the interaction with the medium under ultranarrow $\mathrm{CPO}$ conditions. In particular, we investigate the role of the small population remaining in the excited state, which induces a detrimental additive noise through spontaneous emission.

\section{CLASSICAL BEHAVIOR}

In this section, we first experimentally test the classical model derived in Ref. [27]. A $\Lambda$ system composed of two $\sigma^{+}$ and $\sigma^{-}$transitions is excited by an electric field propagating along the $z$ direction given by

$$
\mathbf{E}(z, t)=\frac{\hbar}{d}\left[\Omega_{D}(z, t) \mathbf{e}_{\|}+g \mathcal{E}(z, t) \mathbf{e}_{\perp}\right] e^{-i \omega_{0}\left(t-\frac{z}{c}\right)}+\text { H.c. },
$$

where $\Omega_{D}$ is the Rabi frequency of the monochromatic driving pump field at frequency $\omega_{0}$ and $\mathcal{E}$ the dimensionless envelope of the weaker field that we want to store. The two fields can oscillate at two different optical frequencies, since $\mathcal{E}$ can be time dependent in a frame rotating at $\omega_{0}$ (see Appendix A for the details of the notations). The quantity $g=d \sqrt{\omega_{0} / 2 \hbar \epsilon_{0} V}$ holds for the atom-light dipolar coupling strength, where $d$ is the transition dipole moment and $V$ the field quantization volume.

In such a system, the transmission of a classical input probe field depends on its relative phase $\Theta$ with respect to the pump field. If the probe spectrum is symmetric with respect to the pump frequency $\omega_{0}$ and fits within the CPO linewidth, the phase-sensitive transmission coefficients $T_{\Theta=0}$ and $T_{\Theta=\frac{\pi}{2}}$ are given by [27]

$$
\begin{aligned}
T_{\frac{\pi}{2}} & =\exp \left[\frac{g^{2} N}{2 \Gamma c} \int_{0}^{L} d z\left(\frac{2 s(z)}{\frac{\gamma_{t}}{\Gamma_{0}}+3 s(z)}-1\right) \frac{1}{1+3 s(z)}\right], \\
T_{0} & =\exp \left[-\frac{g^{2} N}{2 \Gamma c} \int_{0}^{L} \frac{d z}{1+3 s(z)}\right],
\end{aligned}
$$

where $N$ is the number of atoms interacting with the field, $\gamma_{t}$ the transit-induced decay and feeding rate of the lower-level population, $\Gamma_{0}$ the spontaneous emission decay rate of the upper level, and $\Gamma$ the optical coherence decay rate. The saturation parameter of the transitions $s(z)=\Omega_{D}^{2}(z) / \Gamma \Gamma_{0}$ is $z$ dependent, because of the absorption of the pump along propagation [see Eq. (19) and Appendix B for more details]. Although $T_{0}$ is the usual nonlinear absorption of a saturated transition, $T_{\frac{\pi}{2}}$ has a more complex shape, because of the ultranarrow $\mathrm{C}^{2} \mathrm{PO}$ contribution.

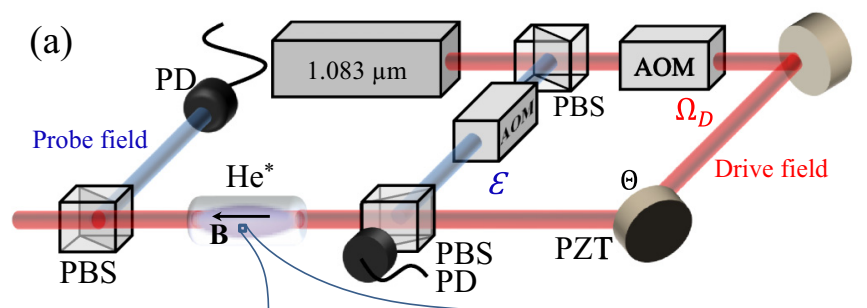

(b)

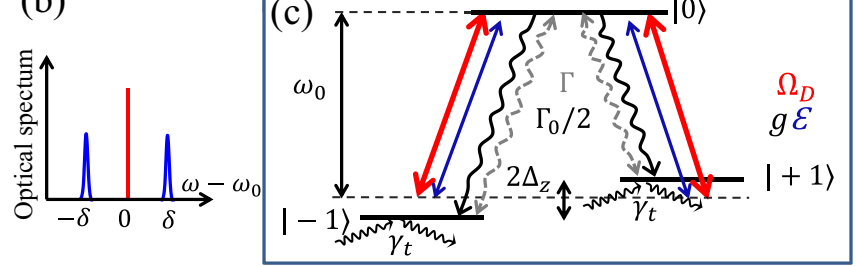

FIG. 1. (a) Experimental setup. The laser is split by a polarizing beam splitter (PBS) to obtain the orthogonally polarized pump $\left(\Omega_{D}\right.$, red) and probe $(\mathcal{E}$, blue) fields. Their temporal and spectral shapes are controlled using acousto-optic modulators (AOMs): The probe spectrum (b) has two components symmetrically detuned by $\pm \delta$ from the coupling frequency. The relative phase $\Theta$ between the probe and pump fields is scanned with a mirror mounted on a piezoelectric actuator (PZT), and measured at the entrance of the cell. At the cell output, a third PBS isolates the probe from the driving field before detection. (c) Level structure. The $2{ }^{3} S_{1} \leftrightarrow 2{ }^{3} P_{1}$ transition excited by linearly polarized light ends up in a closed $\Lambda$ structure [18]. $\Gamma_{0}, \Gamma$, and $\gamma_{t}$ are the decay rates of the excited-state population, the optical coherences, and the lower-level populations, respectively.

To check this model, we measure the transmission of a weak classical probe field under such ultranarrow CPO conditions, using the setup described in Fig. 1(a). Since we need the pump and probe fields to be coherent for the CPO process to occur, they are both derived from the same 2$\mathrm{MHz}$ linewidth Eagleyard distributed feedback (DFB) laser. They are separated by a polarizing beam splitter (PBS), and two different acousto-optic modulators (AOMs) allow one to independently control their amplitudes and frequencies. Here, we investigate the situation depicted in Fig. 1(b) where the probe spectrum consists of two tones, called the signal and idler, respectively detuned by $+\delta$ and $-\delta$ with respect to the pump field. The relative phase $\Theta$ between the probe and pump fields is scanned owing to a mirror mounted on a piezoelectric actuator (PZT) and placed in the path of the pump field. A second PBS recombines the fields at the entrance of the helium cell. A small part of the fields, exiting the other port of the PBS, is detected to monitor $\Theta$. After propagation in the 6-cm-long cell filled with 1 Torr of helium, the probe field is isolated from the pump field by a third PBS before detection. The cell is protected from stray magnetic fields by a $\mu$-metal shield, and a longitudinal magnetic field is applied to lift the degeneracy between the Zeeman sublevels. Inside the cell, the $1 / e^{2}$ waists of the drive and probe beams are $2 \mathrm{~mm}$.

Figure 1(c) shows the excitation scheme of the $2{ }^{3} S_{1} \leftrightarrow$ $2^{3} P_{1}$ transition of metastable helium. The quantization axis of the atomic levels is chosen along the propagation direction of light. The excited and ground levels are composed of three Zeeman sublevels. Since the transition $m=0 \leftrightarrow m=0$ is forbidden when the levels have the same total momentum 


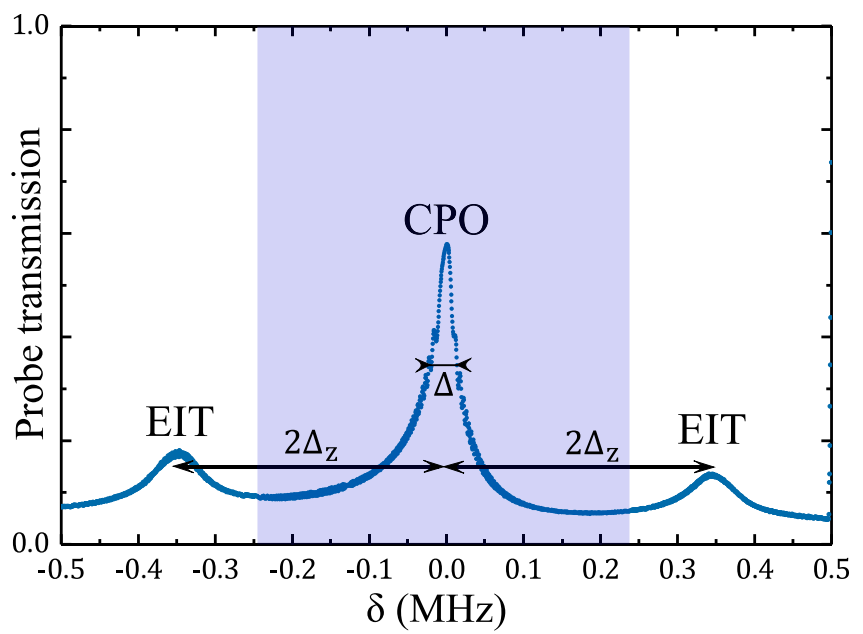

FIG. 2. Measurement of a $100-\mu \mathrm{W}$ probe field absorption spectrum under CPO conditions, in the presence of a $10-\mathrm{mW}$ driving field. Within the nearly 1-GHz Doppler-broadened absorption width of the transition, three transmission resonances are visible. A small longitudinal magnetic field shifts the EIT resonances $\pm 2 \Delta_{z}$ away from the central CPO resonance. In our experimental conditions, the spectrum of the signal is fully included within the shadowed region, where no Raman coherence is excited.

$J$, the system ends up in a closed $\Lambda$ scheme composed of the $m= \pm 1$ ground states and the $m=0$ excited state [29]. One has experimentally $\Gamma / 2 \pi \simeq 0.8 \mathrm{GHz}, \Gamma_{0} / 2 \pi=$ $1.6 \mathrm{MHz}$, and $\gamma_{t} / 2 \pi \simeq 20 \mathrm{kHz}$.

The total field $\mathbf{E}$ can be decomposed in the circular basis $\left\{\mathbf{e}_{+}, \mathbf{e}_{-}\right\}$using

$$
\mathbf{E} \cdot \mathbf{e}_{ \pm}=\frac{\mathbf{E} \cdot \mathbf{e}_{\|} \pm i \mathbf{E} \cdot \mathbf{e}_{\perp}}{\sqrt{2}}
$$

where $\mathbf{e}_{\|}$and $\mathbf{e}_{\perp}$ are the crossed linear polarization directions of the pump and probe fields, respectively. The system thus experiences balanced excitations along the two legs of the $\Lambda$ system: The $m=-1 \leftrightarrow m=0(m=1 \leftrightarrow m=0)$ transition is excited by a beat note due to the $\sigma^{+}\left(\sigma^{-}\right)$components of the probe and pump fields, so that CPOs occur between the $|-1\rangle(|1\rangle)$ ground state and the $|0\rangle$ excited state. Depending on the relative polarization angle and on the relative phase $\Theta$ between the two fields, symmetric or antisymmetric combinations of CPOs between both legs of the system can be excited. In particular, when the two fields are orthogonally polarized, a phase difference $\Theta=\pi / 2$ modulo $\pi$ excites the antisymmetric mode, in which the two CPO phenomena are in antiphase. The population then oscillates between the two ground states of the system. This also leads to an ultranarrow transparency window for the two-frequency probe, centered on the pump field frequency (see Fig. 2). On the contrary, when $\Theta=0$, only probe absorption remains.

For degenerate Zeeman ground states, the pump (probe) field exciting the left leg of the $\Lambda$ system and the probe (pump) field along the right leg lead to a two-photon EIT resonance when both light fields have the same frequency. This also corresponds to the situation where the CPO resonance condition is fulfilled. In order to get rid of EIT two-photon resonance, we apply a longitudinal magnetic field to the atoms. Then, the

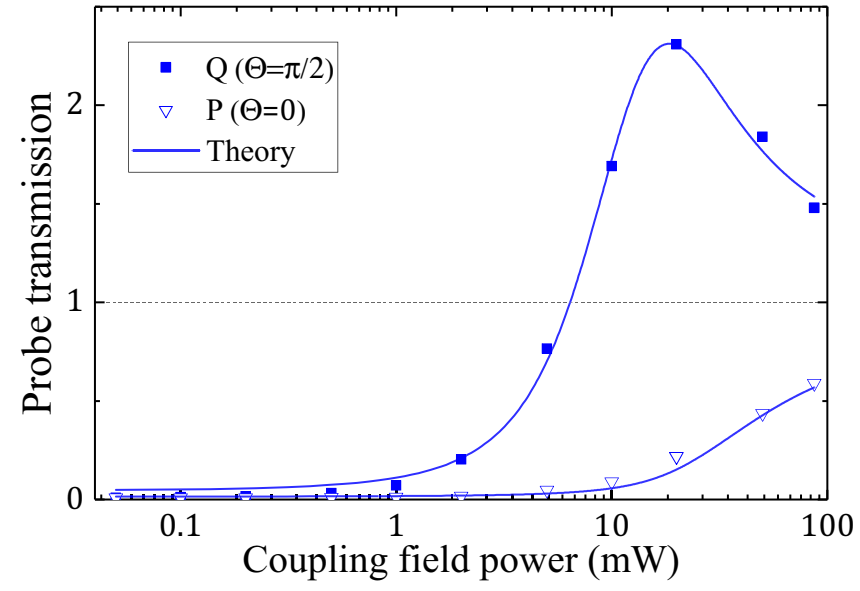

FIG. 3. Measured evolution of the probe field transmission vs pump field power. Solid squares (open triangles): $\Theta=\pi / 2(\Theta=$ $0 \mathrm{~s})$. The input probe field contains two spectral components at $v= \pm 2 \mathrm{kHz}$, with equal amplitudes. Solid line: Theory based on Eqs. (2) taking into account the $\sim 20 \%$ residual absorption from the $2{ }^{3} S_{1} \leftrightarrow 2{ }^{3} P_{2}$ transition.

Zeeman shift $2 \Delta_{z}$ between the $m= \pm 1$ ground-state sublevels restrains Raman coherence from being excited, provided the probe spectrum fits within a window of width smaller than $4 \Delta_{z}-W_{\mathrm{EIT}}$, where $W_{\mathrm{EIT}}$ is the EIT linewidth. Figure 2 shows an experimental transmission spectrum of a single-frequency probe field in such conditions: In the center of the nearly 1-GHz Doppler broadened absorption window, one can see a CPO resonance surrounded by two EIT resonances, shifted because of the Zeeman shift. When the probe spectrum is fully confined to the shadowed region of this plot, EIT is avoided and only ultranarrow CPO occurs.

Figure 3 shows a comparison between the theoretical transmission coefficients for the two field quadratures $P$ and $Q$ obtained from Eqs. (2) with $\Theta=0$ and $\pi / 2$, respectively, and the experimental data. An excellent agreement is observed. Below an input optical pump field power of $\sim 3 \mathrm{~mW}$, ultranarrow CPOs cannot be excited because the saturation induced by the pump field is too weak. Above an input optical pump power of $\sim 30 \mathrm{~mW}$, the strong saturation of the atoms by the pump makes the medium transparent for the probe field and prevents the $\mathrm{CPO}$ resonance from appearing. In between these two regimes, the CPO is efficiently excited and a strong phase-sensitive behavior takes place.

Equations (2) and (19) allow us to extract fitting functions (solid lines in Fig. 3), which are explicitly given in Appendix B and which involve four fitting coefficients. The first one corresponds to the optical depth of the medium $g^{2} N L / 2 \Gamma c$, whose fitted value of $2.8(1)$ is consistent with measurements obtained in previous works $[18,30]$. The second parameter is $\gamma_{t} / \Gamma_{0}=9.6(9) \times 10^{-2}$. It is one order of magnitude larger than the value extracted from the width of the CPO resonance of Fig. 2. We attribute this discrepancy to the existence of residual absorption by the $D_{2}$ line, which reduces the $\mathrm{CPO}$ efficiency, leading to a somewhat larger effective $\gamma_{t}$. This $20(2) \%$ residual absorption by the $D_{2}$ transition is taken into account as another fitting parameter. It is a few percent larger than the residual absorption 
measured independently [30], but consistent with a larger saturation broadening. The last coefficient is given by $s / P_{\mathrm{opt}}=4.7(5) \times 10^{-1} \mathrm{~W}^{-1}$.

As shown in Ref. [27], we recall that an input probe field spectrum symmetric with respect to the pump keeps this symmetry along propagation. However, a monochromatic input probe field spectrum detuned by $+\delta$ from the pump results in the generation of another probe component detuned by $-\delta$ along propagation. This spontaneous idler field generation suggests that $\mathrm{CPO}$ is a multiphoton process, as was initially derived by Boyd [21], coherently exchanging pairs of photons from the bichromatic probe towards the monochromatic pump, and conversely, depending on their global relative phase. The experimental results of Fig. 3 confirm the phase sensitivity of the process. However, the quantum nature of $\mathrm{CPO}$ is a subject of debate because of the possible classical description of its main features, as only populations are explicitly involved for the description of the phenomenon. The calculation developed in Sec. III puts an end to this uncertainty by dealing with the quantum features of CPO, thus exploring a phenomenon at the frontier between classical and quantum optics.

\section{QUANTUM TREATMENT OF THE PROBE PROPAGATION}

Let us now study the evolution of the quantum properties of the probe field along propagation in the presence of a steady-state pump. The goal of this quantum treatment is to investigate whether CPO-based protocols can be considered for quantum applications. To this aim, we need to explicitly consider the quantum fluctuations inherent in the atomic dynamics, and their effects on light noise correlations. For the sake of simplicity, we only consider the effect of the intrinsic noise sources, i.e., spontaneous emission (so that $\Gamma=\Gamma_{0} / 2$ and $\left.\gamma_{t}=0\right)$.

We develop the spectral complex Fourier amplitudes of the probe field operator $\mathcal{E}(z, t)$ and of its Hermitian conjugate $\mathcal{E}(z, t)^{\dagger}$,

$$
\begin{aligned}
\mathcal{E}(z, v) & =\frac{1}{\sqrt{2 \pi}} \int_{-\infty}^{+\infty} \mathcal{E}(z, t) e^{i v t} d t, \\
\mathcal{E}^{\dagger}(z, v) & =\frac{1}{\sqrt{2 \pi}} \int_{-\infty}^{+\infty} \mathcal{E}(z, t)^{\dagger} e^{i v t} d t,
\end{aligned}
$$

where we voluntarily use the same notation for the timedependent probe field and its Fourier components to avoid cumbersome notations. The two functions are clearly distinguished by their arguments. It is important to notice that $\mathcal{E}(z, v)$ and $\mathcal{E}^{\dagger}(z, v)$ are not Hermitian conjugates of each other.

The equations of evolution are derived in the Heisenberg picture but, to avoid clumsy notations, the time dependence is not explicitly written.

\section{A. Evolution of the atoms}

We consider an ensemble of atoms described by a $\Lambda$ scheme similar to the one of Fig. 1(c). Two ground states $|-1\rangle$ and $|+1\rangle$ are optically coupled to the same excited state $|e\rangle$, with both transitions having the same frequency $\omega_{0}$. The atom $j$ is described by the set of operators $\sigma_{\mu \nu}^{j}$, defined in the frame rotating at $\omega_{0}$ as

$$
\sigma_{\nu v}^{j}=|v\rangle_{j j}\langle\nu| \text { and } \sigma_{ \pm 1 \mp 1}^{j}=| \pm 1\rangle_{j j}\langle\mp 1|,
$$

where $v \in\{e,-1,+1\}$ and, for the optical coherences,

$$
\sigma_{ \pm 1 e}^{j}=| \pm 1\rangle_{j j}\langle e| e^{i \omega_{0}(t-z / c)} .
$$

When a longitudinal magnetic field is applied along the $z$ axis and when the pump and probe fields propagate into the medium, the Hamiltonian in the rotating-wave approximation is $\mathrm{H}^{j}=\mathrm{H}_{\mathcal{Z}}^{j}+\mathrm{H}_{d}^{j}$, where

$$
\mathrm{H}_{\mathcal{Z}}^{j}=\hbar \Delta_{z}\left(\sigma_{11}^{j}-\sigma_{-1-1}^{j}\right)
$$

corresponds to the Zeeman interaction with the magnetic field, shifting the ground states by $\pm \Delta_{z}$, and

$$
\mathrm{H}_{d}^{j}=\hbar\left(\sigma_{e 1}^{j} \mathbf{e}_{-}+\sigma_{e-1}^{j} \mathbf{e}_{+}\right)\left(\Omega_{D} \mathbf{e}_{\|}+g \mathcal{E} \mathbf{e}_{\perp}\right)+\text { H.c. }
$$

is the electric-dipole interaction with the optical fields.

Rather than considering individual atomic operators, we assume that the medium is homogeneous and define continuous $z$-dependent operators $\sigma_{\mu \nu}(z)$ by averaging the density operator components over a thin slice of medium $\mathcal{T}(z)$ containing $N$ atoms,

$$
\sigma_{\mu \nu}(z, t)=\frac{1}{N} \sum_{j \in \mathcal{T}(z)} \sigma_{\mu \nu}^{j}(t) .
$$

This approximation is valid when the width of the slice is large enough to contain a large number of atoms, but small enough compared to the light wavelength, so that it is possible to differentiate on $z$. The Heisenberg-Langevin equations, which govern the dynamics of the atomic operators $\sigma_{\mu \nu}$, are then [31]

$$
\frac{\partial}{\partial t} \sigma_{\mu \nu}=\frac{1}{i \hbar}\left[\sigma_{\mu \nu}, \mathrm{H}\right]+\mathcal{R}\left(\sigma_{\mu \nu}\right)+F_{\mu \nu},
$$

where $\mathrm{H}$ is the sum of the $\mathrm{H}^{j}$ 's in the slice $\mathcal{T}(z), \mathcal{R}$ is the spontaneous emission dissipator, and $F_{\mu \nu}$ are the Langevin forces, i.e., quantum fluctuations originating from the coupling of the atoms to the vacuum electromagnetic bath. These time-dependent operators are spatially averaged using

$$
F_{\mu \nu}(z, t)=\frac{1}{N} \sum_{j \in \mathcal{T}(z)} F_{\mu \nu}^{j}(t) .
$$

The average of the Langevin forces is zero, and we assume that their correlation timescale can be neglected with respect to the timescales of the dynamics of the system, so that

$$
\left\langle F_{\mu \nu}\left(z_{1}, t_{1}\right) F_{\alpha \beta}\left(z_{2}, t_{2}\right)\right\rangle=\frac{\delta_{z_{1}}^{z_{2}}}{N} D_{\mu \nu}^{\alpha \beta} \delta\left(t_{1}-t_{2}\right),
$$

where $\delta_{z_{1}}^{z_{2}}$ is a Kronecker symbol equal to zero for two different spatial positions, and $D_{\mu \nu}^{\alpha \beta}$ is a diffusion coefficient, given by the Einstein generalized relations [31]

$$
D_{\mu \nu}^{\alpha \beta}=\left\langle\mathcal{R}\left(\sigma_{\mu \nu} \sigma_{\alpha \beta}\right)-\sigma_{\mu \nu} \mathcal{R}\left(\sigma_{\alpha \beta}\right)-\sigma_{\alpha \beta} \mathcal{R}\left(\sigma_{\mu \nu}\right)\right\rangle .
$$

\section{B. Evolution of the optical fields}

The total optical field $\mathbf{E}$ propagates inside the medium according to Maxwell's equations in the slowly varying envelope approximation, as derived in Ref. [32]. It then is possible 
to obtain separate equations for the pump and probe field envelopes by projecting the equation for the total field on their respective orthogonal polarization directions using Eqs. (1) and (3), leading to

$$
\begin{aligned}
\left(c \partial_{z}+\partial_{t}\right) \Omega_{D}(z, t) & =i g^{2} N\left(\sigma_{e 1}+\sigma_{e-1}\right), \\
\left(c \partial_{z}+\partial_{t}\right) \mathcal{E}(z, t) & =g N\left(\sigma_{e 1}-\sigma_{e-1}\right) .
\end{aligned}
$$

As shown in Ref. [27], the quadrature operators of the complex amplitude $\mathcal{E}$, defined by (see Appendix A for more details)

$$
\begin{aligned}
& \mathcal{P}(z, t)=\frac{1}{2}\left[\mathcal{E}(z, t)+\mathcal{E}(z, t)^{\dagger}\right], \\
& \mathcal{Q}(z, t)=\frac{1}{2 i}\left[\mathcal{E}(z, t)-\mathcal{E}(z, t)^{\dagger}\right],
\end{aligned}
$$

are the relevant quantities to describe the probe field. If the probe field is monochromatic at the frequency of the pump $\omega_{0}$, then these quantities coincide with the usual definition of the probe quadratures. More generally, these quantities match the usual definition of the quadratures of the probe field as soon as its spectrum is symmetric with respect to the pump frequency. This is, for instance, the case in the situation of a bichromatic probe field detuned by $\pm \delta$ from the pump. Then the transmission coefficients of Eqs. (2) are those of $\mathcal{Q}$ and $\mathcal{P}$, respectively, derived from the classical calculation of Ref. [27]. However, in the situation of a monochromatic probe field different from $\omega_{0}$ or any other nonsymmetric spectrum with respect to the pump, $\mathcal{P}$ and $\mathcal{Q}$ are nontrivial time-dependent mixtures of the probe field components.

\section{Linearization}

The preceding equations can be solved using a perturbative approach at first order in quantum probe field. Any observable $\mathcal{O}$ can then be expanded as follows,

$$
\mathcal{O}=\langle\mathcal{O}\rangle_{0}+\langle\mathcal{O}\rangle_{1}+\delta \mathcal{O},
$$

where $\langle\mathcal{O}\rangle_{0}$ stands for the mean value of $\mathcal{O}$ in the presence of the classical pump field alone, $\langle\mathcal{O}\rangle_{1}$ is the first-order perturbation due to the presence of the probe field, and $\delta \mathcal{O}$ represents the linearized quantum fluctuation part of $\mathcal{O}$.

Notice that we neglect the influence of the probe field on the evolution of the pump field, and we assume that the evolution of the quantum fluctuations of any operator is governed by the dynamics generated by the pump field only.

\section{RESULTS}

\section{A. Zeroth order}

The zeroth-order dynamics is obtained from the mean values of Eqs. (10) and (14) in the steady-state regime in the presence of the pump alone, reading

$$
\begin{aligned}
0 & =\frac{1}{i \hbar}\left[\left\langle\sigma_{\mu \nu}\right\rangle_{0},\langle\mathrm{H}\rangle_{0}\right]+\mathcal{R}\left(\left\langle\sigma_{\mu \nu}\right\rangle_{0}\right), \\
c \partial_{z} \Omega_{D} & =i g^{2} N\left(\left\langle\sigma_{e 1}\right\rangle_{0}+\left\langle\sigma_{e-1}\right\rangle_{0}\right) .
\end{aligned}
$$

The time dependence is skipped because the pump and the system are assumed to be steady.
Assuming that the pump Rabi frequency is real, the density matrix of the system is then given by

$$
\langle\sigma\rangle_{0}=\left(\begin{array}{ccc}
\frac{s}{1+3 s} & \frac{i \Omega_{D}}{\sqrt{2} \Gamma_{0}(1+3 s)} & \frac{i \Omega_{D}}{\sqrt{2} \Gamma_{0}(1+3 s)} \\
-\frac{i \Omega_{D}}{\sqrt{2} \Gamma_{0}(1+3 s)} & \frac{1+2 s}{2+6 s} & 0 \\
-\frac{i \Omega_{D}}{\sqrt{2} \Gamma_{0}(1+3 s)} & 0 & \frac{1+2 s}{2+6 s}
\end{array}\right) .
$$

Such a density matrix merely describes the usual saturation of the transition, leading to a nonzero amount of population in the excited state. The absorption of the pump leads to a $z$-dependent $s$ parameter, which obeys the following equation,

$$
\partial_{z} s=-\frac{2 g^{2} N}{c \Gamma_{0}} \frac{s}{1+3 s},
$$

where $2 g^{2} N L / c \Gamma_{0}$ is the optical depth of the medium in the linear absorption regime.

\section{B. First order: Expectation value}

Equations (10) and (14) in the Fourier domain give the following set of equations for the probe field expectation value,

$$
\begin{aligned}
-i \nu\left\langle\sigma_{\mu \nu}(z, \nu)\right\rangle_{1}= & \frac{1}{i \hbar}\left(\left[\left\langle\sigma_{\mu \nu}\right\rangle_{1},\langle\mathrm{H}\rangle_{0}\right]+\left[\left\langle\sigma_{\mu \nu}\right\rangle_{0},\langle\mathrm{H}\rangle_{1}\right]\right) \\
& +\mathcal{R}\left(\left\langle\sigma_{\mu \nu}\right\rangle_{1}\right), \\
\left(c \partial_{z}-i v\right)\langle\mathcal{E}(z, v)\rangle= & g N\left(\left\langle\sigma_{e 1}\right\rangle_{1}+\left\langle\sigma_{e-1}\right\rangle_{1}\right) .
\end{aligned}
$$

When the probe field spectrum fits within the $4 \Delta_{z}-W_{\text {EIT }}$ window centered on $\omega_{0}$, as shown in Fig. 2 (assuming $v \ll$ $\left.\Delta_{z} \ll \Gamma\right)$, the probe field complex amplitude propagates according to

$$
\left(c \partial_{z}-i v\right)\langle\mathcal{E}(z, v)\rangle=\frac{g^{2} N}{\Gamma_{0}(1+3 s)} \frac{i v\langle\mathcal{E}\rangle-\Delta\left\langle\mathcal{E}^{\dagger}\right\rangle}{\Delta-i v},
$$

where $\Delta=2 \Omega_{D}^{2}(z) / \Gamma_{0}=s(z) \Gamma_{0}$ is the saturation-broadened CPO linewidth. Because of the pump absorption, the CPO linewidth $\Delta$ decreases during propagation along $z$. Such an equation can be rewritten using the Fourier components of the quadratures $\mathcal{P}(z, v)$ and $\mathcal{Q}(z, v)$,

$$
\begin{aligned}
& \partial_{z}\langle\mathcal{Q}\rangle(z, v)=\left(\Lambda_{1}(z, v)+i \frac{v}{c}\right)\langle\mathcal{Q}(z, v)\rangle, \\
& \partial_{z}\langle\mathcal{P}(z, v)\rangle=\left(\Lambda_{2}(z, v)+i \frac{v}{c}\right)\langle\mathcal{P}(z, v)\rangle,
\end{aligned}
$$

with $\Lambda_{1,2}$ given by

$$
\begin{aligned}
& \Lambda_{1}(z, v)=+\frac{g^{2} N}{c \Gamma_{0}(1+3 s)} \frac{\Delta+i v}{\Delta-i v}, \\
& \Lambda_{2}(z, v)=-\frac{g^{2} N}{c \Gamma_{0}(1+3 s)} .
\end{aligned}
$$

Equations (22) do not mix $\mathcal{P}(z, v)$ and $\mathcal{Q}(z, v)$, which are thus eigenmodes for the propagation. Compared to Ref. [27], the equations are now derived without adiabatic expansion, and with spontaneous emission as the only noise source. The classical CPO dispersion behavior can thus be obtained by an 
adiabatic expansion of $\Lambda_{1}$,

$$
\begin{aligned}
\Lambda_{1}+i \frac{\nu}{c} \simeq & \frac{g^{2} N}{c \Gamma_{0}(1+3 s)}+i v \frac{1+\frac{g^{2} N}{\Omega_{D}^{2}(1+3 s)}}{c} \\
& -\frac{g^{2} N}{c \Gamma_{0}(1+3 s)} \frac{v^{2}}{\Delta^{2}} .
\end{aligned}
$$

From Eqs. (22) and (23), we can see that CPOs generate a phase-sensitive behavior as soon as the spectrum of the probe fits within the CPO linewidth $v \ll \Delta$ : The first term of Eq. (24) corresponds to the amplification, the second one corresponds to the associated decrease in group velocity, and the third term limits the bandwidth for which these effects are efficient. In the limit of an ideal system $\left(\gamma_{t} \rightarrow 0\right.$ and $\Gamma=\Gamma_{0} / 2$ ), the adiabatic part of the $\Lambda_{i}$ 's functions match with the quantities derived classically in the integrals of Eqs. (2).

We now suppose that the probe spectrum is well within the CPO linewidth $\Delta$, with $v \ll \Delta$. Then, integrating Eq. (22) and using Eqs. (19) and (23), one can find the expectation values of the quadratures after propagation,

$$
\begin{aligned}
& \langle\mathcal{Q}(z, v)\rangle=\sqrt{G(z)} e^{\frac{i v z}{c}}\langle\mathcal{Q}(0, v)\rangle, \\
& \langle\mathcal{P}(z, v)\rangle=\frac{1}{\sqrt{G(z)}} e^{i \frac{v z}{c}}\langle\mathcal{P}(0, v)\rangle,
\end{aligned}
$$

where $1 / G(z)=s(z) / s(0)<1$ corresponds to the decrease of pump intensity due to absorption. The phase-sensitive transmission of the probe field is here visible, leading to an increasing (decreasing) amplitude for the quadrature $\mathcal{Q}(\mathcal{P})$.

\section{First order: Fluctuations}

The linearized equations of evolution for the fluctuation of the operators can be deduced from Eqs. (10) and (14) in the Fourier domain,

$$
\begin{aligned}
-i \nu \partial_{t} \delta \sigma_{\mu \nu}(z, v)= & \frac{1}{i \hbar}\left(\left[\delta \sigma_{\mu \nu},\langle\mathrm{H}\rangle_{0}\right]+\left[\left\langle\sigma_{\mu \nu}\right\rangle_{0}, \delta \mathrm{H}\right]\right) \\
& +\mathcal{R}\left(\delta \sigma_{\mu \nu}\right)+F_{\mu \nu}, \\
\left(c \partial_{z}-i v\right) \delta \mathcal{E}(z, v)= & g N\left(\delta \sigma_{e 1}+\delta \sigma_{e-1}\right) .
\end{aligned}
$$

These equations are valid at first order and describe the effect of the pump saturation on the probe fluctuations. They neglect any effect of the probe evolution itself on its own fluctuations. The quadrature fluctuations are then given by

$$
\begin{aligned}
& \partial_{z} \delta \mathcal{Q}(z, v)=\left(\Lambda_{1}+i \frac{v}{c}\right) \delta \mathcal{Q}(z, v)+\sum_{\mu \nu} \alpha_{\mu \nu} F_{\mu \nu}, \\
& \partial_{z} \delta \mathcal{P}(z, v)=\left(\Lambda_{2}+i \frac{v}{c}\right) \delta \mathcal{P}(z, v)+\sum_{\mu \nu} \beta_{\mu \nu} F_{\mu \nu},
\end{aligned}
$$

with

$$
\begin{aligned}
\sum_{\mu \nu} \alpha_{\mu \nu} F_{\mu \nu}= & \frac{-g N}{\sqrt{2} c \Gamma_{0} \Delta}\left[v\left(F_{e-1}-F_{e 1}-F_{-1 e}+F_{1 e}\right)\right. \\
& \left.+\sqrt{2} \Omega_{D}\left(F_{11}-F_{-1-1}\right)\right] \\
\sum_{\mu \nu} \beta_{\mu \nu} F_{\mu \nu}= & \frac{+g N}{\sqrt{2} c \Gamma_{0}}\left(F_{e-1}-F_{e 1}+F_{-1 e}-F_{1 e}\right)
\end{aligned}
$$

Three independent combinations of Langevin operators are thus relevant: $F_{\Delta}=F_{11}-F_{-1-1}$ and $F_{ \pm}=F_{e 1} \pm F_{e-1}$. Using Eqs. (22) and (27), it is possible to derive the quadratures after propagation,

$$
\begin{aligned}
\mathcal{Q}(z, \nu)= & \sqrt{G(z) e^{i \frac{i v z}{c}}} \mathcal{Q}(0, v) \\
& +\sum_{\mu \nu} \int_{0}^{z} d x e^{\int_{x}^{z}\left(\Lambda_{1}+i \frac{v}{c}\right) d \xi} \alpha_{\mu \nu} F_{\mu \nu}, \\
\mathcal{P}(z, \nu)= & \frac{1}{\sqrt{G(z)}} e^{i \frac{\nu z}{c}} \mathcal{P}(0, \nu) \\
& +\sum_{\mu \nu} \int_{0}^{z} d x e^{\int_{x}^{z}\left(\Lambda_{2}+i \frac{v}{c}\right) d \xi} \beta_{\mu \nu} F_{\mu \nu} .
\end{aligned}
$$

For each quadrature, the first right-hand side term is just the propagation of the quantum operator with the corresponding gain and accumulated phase, while the second term is the noise accumulated all along the propagation. The quadrature fluctuations after propagation can then be evaluated by computing their squeezing spectra, defined as the Fourier transform of the autocorrelation function. For a quadrature $X$, this spectrum is given by

$$
\begin{aligned}
\mathcal{S}_{X}(z, v) & \equiv \frac{4 c}{L} \int d t e^{i v t}\langle X(z, t) X(z, 0)\rangle \\
& =\frac{4 c}{L} \int d v^{\prime}\left\langle X(z, v) X\left(z, v^{\prime}\right)\right\rangle,
\end{aligned}
$$

where the factor $4 c / L$ is the vacuum shot-noise renormalization factor (see Appendix A).

Applying Eq. (30) to $\mathcal{P}$ and $\mathcal{Q}$ in Eqs. (29) leads to

$$
\begin{aligned}
\mathcal{S}_{\mathcal{P}}(z, v)= & \frac{4 c}{L} \int d v^{\prime}\left(e^{\int_{0}^{z}\left[\Lambda_{2}(v)+\Lambda_{2}\left(v^{\prime}\right)\right] d \xi}\left\langle\mathcal{P}(0, v) \mathcal{P}\left(0, v^{\prime}\right)\right\rangle\right. \\
& +\sum_{a b c d} \int_{0}^{z} \int_{0}^{z} d x d x^{\prime} e^{\int_{x}^{z} \Lambda_{2}(v) d \xi+\int_{x^{\prime}}^{z} \Lambda_{2}\left(v^{\prime}\right) d \xi} \\
& \left.\times \beta_{a b} \beta_{c d}\left\langle F_{a b}(x, v) F_{c d}\left(x^{\prime}, v^{\prime}\right)\right\rangle\right) \\
\mathcal{S}_{\mathcal{Q}}(z, v)= & \frac{4 c}{L} \int d v^{\prime}\left(e^{\int_{0}^{z}\left[\Lambda_{1}(v)+\Lambda_{1}\left(v^{\prime}\right)\right] d \xi}\left\langle\mathcal{Q}(0, v) \mathcal{Q}\left(0, v^{\prime}\right)\right\rangle\right. \\
& +\sum_{a b c d} \int_{0}^{z} \int_{0}^{z} d x d x^{\prime} e^{\int_{x}^{z} \Lambda_{1}(v) d \xi+\int_{x^{\prime}}^{z} \Lambda_{1}\left(v^{\prime}\right) d \xi} \\
& \left.\times \alpha_{a b} \alpha_{c d}\left\langle F_{a b}(x, v) F_{c d}\left(x^{\prime}, v^{\prime}\right)\right\rangle\right)
\end{aligned}
$$

In both equations, the first term simply represents the propagation of the input squeezing spectrum $\mathcal{S}_{X}(0, v)$. The second one is related to the influence of the medium noise correlations $\left\langle F_{\mu \nu}\left(z_{1}, t_{1}\right) F_{\alpha \beta}\left(z_{2}, t_{2}\right)\right\rangle$. The diffusion coefficients, which can be obtained with the generalized Einstein relation [see Eq. (12)], are given in Table I. The $z$ dependence of the variances can then be deduced from the terms of each quadrature squeezing spectrum, using Eqs. (12), (19), 
TABLE I. Diffusion coefficients associated with the correlations of two Langevin operators [see Eq. (12)].

\begin{tabular}{lccc}
\hline \hline$D$ & $\left.\ldots F_{\Delta}(z, v)\right\rangle$ & $\left.\ldots F_{+}(z, v)\right\rangle$ & $\left.\ldots F_{-}(z, v)\right\rangle$ \\
\hline$\left\langle F_{\Delta}(z, v) \ldots\right.$ & $\Gamma_{0} \frac{s}{1+3 s}$ & 0 & 0 \\
$\left\langle F_{+}(z, v) \ldots\right.$ & 0 & 0 & 0 \\
$\left\langle F_{-}(z, v) \ldots\right.$ & 0 & 0 & $\Gamma_{0}$ \\
\hline \hline
\end{tabular}

and (23),

$$
\begin{aligned}
\mathcal{S}_{P}(z, v)= & \frac{1}{G(z)} \mathcal{S}_{P}(0, v)+1-\frac{1}{G(z)}+3 s(z) \ln G(z), \\
\mathcal{S}_{Q}(z, v)= & G(z) \mathcal{S}_{Q}(0, v)-1+G(z) \\
& +\frac{v^{2}}{\Gamma_{0}^{2}} \frac{3 \ln G(z)-\frac{1}{s(0)}+\frac{1}{s(z)}}{s(z)} .
\end{aligned}
$$

These equations demonstrate that some noise is added, whatever the input saturation and the spectrum of the probe field. Indeed, the probe field quadratures couple with nonzero noise correlations because the pump field makes the population of the system incoherently cycle from the ground states to the upper state. The small residual population of the excited state can then decay through spontaneous emission, adding some noise to the probe field quadratures. Figure 4

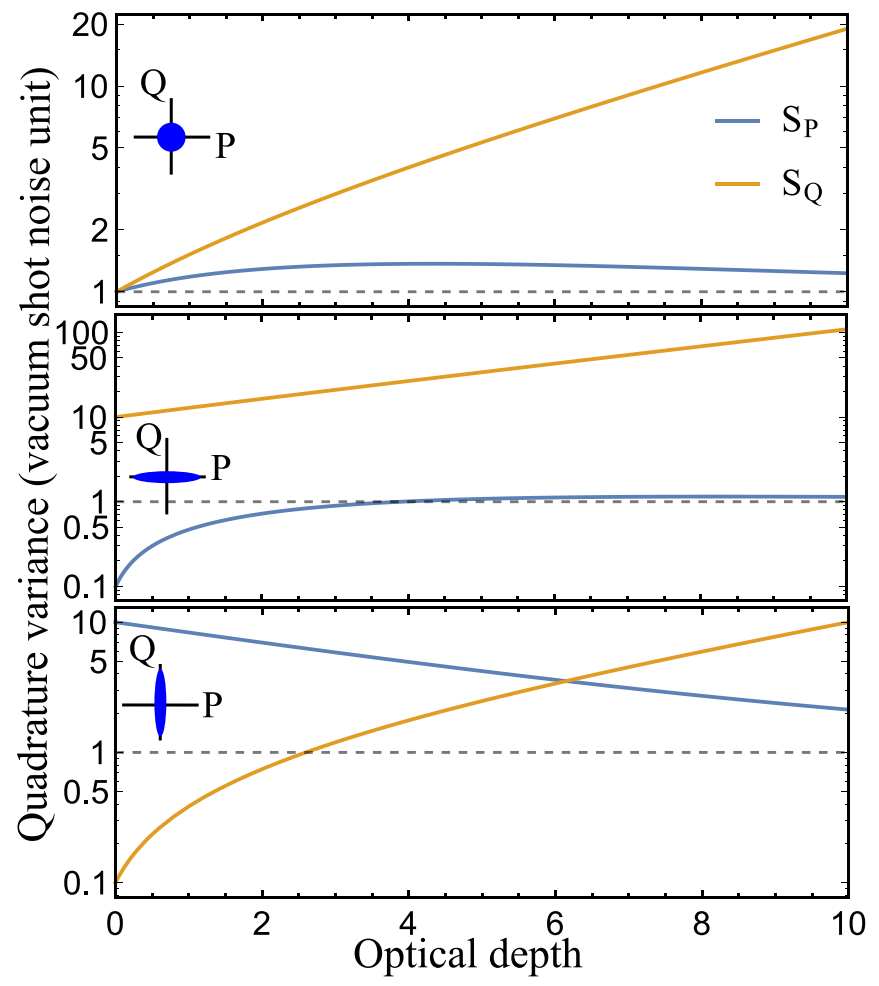

FIG. 4. Evolution of the variances of the quadratures $\mathcal{Q}$ (solid orange line) and $\mathcal{P}$ (dashed blue line) vs medium thickness, for an input saturation level $s(0)=1$ in the case of (a) a shot-noise-limited coherent input state, (b) a $10-\mathrm{dB} \mathcal{P}$-squeezed vacuum input state, and (c) a $10-\mathrm{dB} \mathcal{Q}$-squeezed vacuum input state. The gray dotted-dashed horizontal line corresponds to the standard quantum limit. shows how the noises of both quadratures evolve in the case of a coherent input state or in the case of $\mathcal{P}$-squeezed or $\mathcal{Q}$-squeezed vacuum input states. In all three cases, the $\mathcal{Q}$ component amplification leads to a noise increase. Moreover, the absorption of the $\mathcal{P}$ component makes its noise tend to 1 in the thick medium limit $s(z) \rightarrow 0$. A quantum state propagating in a medium under $\mathrm{CPO}$ conditions thus cannot be preserved and undergoes a nonunitary transformation, which cannot be compensated.

The above calculations are derived through an adiabatic expansion of Eqs. (32), and under the assumptions that spontaneous emission is the only source of noise and that the probe field spectrum fits within the CPO linewidth. The introduction of extra noise sources or the use of broader probe spectra would further increase the noise. But, with the present model, we demonstrate that spontaneous emission alone already forbids CPO from being used for quantum storage. Indeed, the classical description of Ref. [27] evidences the fact that only one quadrature can be stored. Such a classical result might suggest for us to split the two quadratures and store them independently, but this is not allowed by quantum mechanics: The noise added by spontaneous emission is necessary to avoid any violation of Heisenberg inequalities.

\section{CONCLUSION}

In this article, we have investigated the quantum properties of a probe field propagating in an ultranarrow $\mathrm{CPO}$ configuration in a $\Lambda$ system. To this aim, we have treated the probe field quantum mechanically, while keeping a semiclassical approach for the stronger-coupling drive field. Moreover, both the quantum average values and the fluctuations of all quantum observables have been derived analytically at first order in probe field Rabi frequency. We have demonstrated that the small number of atoms that are promoted to the upper level of the $\Lambda$ system leads to spontaneous emission, which is sufficient to destroy the quantum noise properties of the input probe field. We have illustrated this feature by considering several squeezed states of light incident on the medium. In all cases, the variances of the quadratures at the output of the medium exceed the standard quantum limit, showing that squeezing is destroyed.

This conclusion contradicts the statement that phasesensitive amplification automatically generates nonclassical states of light. The CPO phase sensitivity refers here to the dependence on the relative phase between the probe and coupling beams, which is different from the phase-dependent retrieval described in stimulated photon-echo protocols [33,34]. In our system, although the net gain depends on which quadrature is detected, a feature that is reminiscent of phase-sensitive amplifiers, the quadrature whose power decreases with propagation is not "deamplified," but genuinely absorbed.

Moreover, some years ago the investigation of quantum noise properties under slow and fast light propagation [35] showed that for an ideal gain medium the noise figure is always less than two and can be set to 1 , while a loss medium arbitrarily increases the noise because of the random loss of photons. We demonstrate here that in the case of $\mathrm{CPO}$, a quantum noise degradation always arises because of spontaneous emission, even when the transmission is more 
than 1. Indeed, since the CPO phenomenon originates from the saturation of absorption along the two legs of the $\Lambda$ system, it is unavoidably accompanied by a small population in the upper level. Although our initial guess was that this population is so small that it can be neglected for quantum storage using ultranarrow CPOs, it appears that it is sufficient to completely spoil the quantum properties of light. This phenomenon should be kept in mind when using resonant atomic systems to create squeezed light, for example, via quasiresonant four-wave mixing. Although the detuning from resonance might be thought to be large enough to make the excited-level population negligible, one should pay particular attention to the spontaneous emission induced by such small excitation of the system.

\section{ACKNOWLEDGMENT}

The authors acknowledge funding by Indo-French CEFIPRA, Labex PALM, Région IdF DIM Nano-K, and Institut Universitaire de France.

\section{APPENDIX A: LINK BETWEEN ENVELOPE OPERATOR $\mathcal{E}$ AND ANNIHILATION OPERATOR $a$}

This Appendix gives some details about the operator $\mathcal{E}$ used in Eq. (1). $\mathcal{E}$ is the dimensionless complex amplitude of the propagating probe field $\mathbf{E}_{p}$, written in a frame rotating at $\omega_{0}$,

$$
\mathbf{E}_{p}(z, t)=\sqrt{\frac{\hbar \omega_{0}}{2 \epsilon_{0} V}}\left[\mathcal{E}(z, t) e^{-i \omega_{0}\left(t-\frac{z}{c}\right)} \mathbf{e}_{\|}+\text {H.c. }\right] .
$$

In the continuous limit $(V \rightarrow \infty)$, the relation between this envelope operator and the operators acting on the electromagnetic field is [36]

$$
\begin{gathered}
\mathcal{E}(z, t)=+i \sqrt{\frac{L}{2 c \pi}} \int_{-\infty}^{+\infty} a(\omega) e^{-i\left(\omega-\omega_{0}\right)(t-z / c)} d \omega, \\
\mathcal{E}(z, t)^{\dagger}=-i \sqrt{\frac{L}{2 c \pi}} \int_{-\infty}^{+\infty} a^{\dagger}(\omega) e^{+i\left(\omega-\omega_{0}\right)(t-z / c)} d \omega,
\end{gathered}
$$

where $V=L^{3}$ is the quantization volume, and the commutation relation for the electromagnetic field operators is $\left[a\left(\omega_{1}\right), a^{\dagger}\left(\omega_{2}\right)\right]=\delta\left(\omega_{1}-\omega_{2}\right)$. $\mathcal{E}$ and $\mathcal{E}^{\dagger}$ are defined in a frame rotating at $\omega_{0}$, as superpositions of the annihilation and creation operators $a$ and $a^{\dagger}$, respectively. Their spectral components are given by

$$
\begin{gathered}
\mathcal{E}(z, v)=+i \sqrt{\frac{L}{c}} e^{i v z / c} a\left(\omega_{0}+v\right), \\
\mathcal{E}^{\dagger}(z, v)=-i \sqrt{\frac{L}{c}} e^{i v z / c} a^{\dagger}\left(\omega_{0}-v\right) .
\end{gathered}
$$

It should be emphasized that $\mathcal{E}$ and $\mathcal{E}^{\dagger}$ are not Hermitian conjugates of one another. Their commutation rules can be deduced from the field operators $a$ and $a^{\dagger}$,

$$
\left[\mathcal{E}(z, v), \mathcal{E}^{\dagger}\left(z, v^{\prime}\right)\right]=\frac{L}{c} \delta\left(v+v^{\prime}\right) .
$$

The quadratures $\mathcal{P}$ and $\mathcal{Q}$ of $\mathcal{E}$ can then be defined in the Fourier domain by

$$
\begin{aligned}
\mathcal{P}(z, v) & =\frac{1}{2}\left[\mathcal{E}(z, v)+\mathcal{E}^{\dagger}(z, v)\right] \\
& =i \sqrt{\frac{L}{4 c}} e^{i v z / c}\left[a\left(\omega_{0}+v\right)-a^{\dagger}\left(\omega_{0}-v\right)\right], \\
\mathcal{Q}(z, v) & =\frac{1}{2 i}\left[\mathcal{E}(z, v)-\mathcal{E}^{\dagger}(z, v)\right] \\
& =i \sqrt{\frac{L}{4 c}} e^{i v z / c}\left[a\left(\omega_{0}+v\right)+a^{\dagger}\left(\omega_{0}-v\right)\right] .
\end{aligned}
$$

$\mathcal{P}$ and $\mathcal{Q}$ can thus have a complex amplitude.

Using the commutation relation (A4), it is possible to compute the squeezing spectrum for vacuum,

$$
\begin{aligned}
& \int d v^{\prime}\left\langle\mathcal{P}(z, v) \mathcal{P}\left(z, v^{\prime}\right)\right\rangle \\
& =\frac{1}{4} \int d v^{\prime}\left\langle\mathcal{E}^{\dagger}(z, v) \mathcal{E}\left(z, v^{\prime}\right)+\mathcal{E}(z, v) \mathcal{E}^{\dagger}\left(z, v^{\prime}\right)\right\rangle \\
& =\left(\frac{L}{4 c}+\frac{1}{2} \int d v^{\prime}\left\langle\mathcal{E}^{\dagger}(z, v) \mathcal{E}\left(z, v^{\prime}\right)\right\rangle\right) \\
& =\frac{L}{4 c},
\end{aligned}
$$

so that the Eq. (30) leads to $\mathcal{S}_{X}(\omega)=1$.

\section{APPENDIX B: TRANSMISSION COEFFICIENT DEFINITIONS}

\section{Transmission in Eqs. (2)}

The transmission coefficients $T_{\Theta=0, \frac{\pi}{2}}$ are considered after propagation in the whole medium. They are thus related to the $4 \times 4$ transfer matrix per unit length $\mathcal{T}(z)$ given in Eq. (10) of Ref. [27] by

$$
\begin{aligned}
& T_{\Theta=0}=\exp \left[\int_{0}^{L} d z \mathcal{T}_{44}(z)\right], \\
& T_{\Theta=\frac{\pi}{2}}=\exp \left[\int_{0}^{L} d z \mathcal{T}_{33}(z)\right] .
\end{aligned}
$$

Following Refs. [29,37], the Doppler broadening is taken into account by replacing the optical coherence decay rate $\Gamma$ by the Doppler broadening width.

\section{Fitting functions of Fig. 3}

Equations (2) and (19) allow one to extract analytical expressions for the fitting functions of Fig. 3. From Eq. (19) one can obtain

$$
\ln \left(\frac{s(L)}{s(0)}\right)+3 \alpha\left(\frac{s(L)}{s(0)}-1\right) P_{\mathrm{opt}}=-\beta L,
$$


where $\alpha=s / P_{\mathrm{opt}}$ and $\beta=2 g^{2} N / \Gamma_{0}$. This equation can be rewritten

$$
u \frac{s(L)}{s(0)} e^{u \frac{s(L)}{s(0)}}=e^{u-\beta L} u,
$$

with $u=3 \alpha P_{\mathrm{opt}}$. Using the branch 0 of the semianalytical Lambert function $W$, it is then possible to obtain $s(L)$,

$$
s(L)=s(0) \frac{W\left(e^{u-\beta L} u\right)}{u} .
$$

The integration of Eqs. (2) in terms of $s(L) / s(0)$ then gives the following fitting functions,

$$
\begin{gathered}
T_{\Theta=0}=\frac{W\left(e^{u-\beta L} u\right)}{u} \delta, \\
T_{\Theta=\frac{\pi}{2}}=\left(\frac{\frac{u}{3}+\gamma}{\frac{W\left(e^{u-\beta L} u\right)}{3}+\gamma}\right)^{2} \frac{W\left(e^{u-\beta L} u\right)}{u} \delta,
\end{gathered}
$$

where $u$ (and thus $\alpha$ ), $\beta, \gamma_{t} / \Gamma_{0}$ are fitting parameters. Another parameter $\delta$ was added to take into account the residual absorption by the far-detuned $D_{2}$ transition.
[1] A. I. Lvovsky, B. C. Sanders, and W. Tittel, Nat. Photonics 3, 706 (2009).

[2] S. E. Harris, J. E. Field, and A. Imamoglu, Phys. Rev. Lett. 64, 1107 (1990).

[3] M. D. Lukin, S. F. Yelin, and M. Fleischhauer, Phys. Rev. Lett. 84, 4232 (2000).

[4] C. Liu, Z. Dutton, C. H. Behroozi, and L. V. Hau, Nature (London) 409, 490 (2001).

[5] D. F. Phillips, A. Fleischhauer, A. Mair, R. L. Walsworth, and M. D. Lukin, Phys. Rev. Lett. 86, 783 (2001).

[6] K. F. Reim, P. Michelberger, K. C. Lee, J. Nunn, N. K. Langford, and I. A. Walmsley, Phys. Rev. Lett. 107, 053603 (2011).

[7] M. Albert, A. Dantan, and M. Drewsen, Nat. Photonics 5, 633 (2011).

[8] J. J. Longdell, E. Fraval, M. J. Sellars, and N. B. Manson, Phys. Rev. Lett. 95, 063601 (2005).

[9] M. D. Eisaman, A. André, F. Massou, M. Fleischhauer, A. S. Zibrov, and M. D. Lukin, Nature (London) 438, 837 (2005).

[10] J. Appel, E. Figueroa, D. Korystov, M. Lobino, and A. I. Lvovsky, Phys. Rev. Lett. 100, 093602 (2008).

[11] M. Fleischhauer and M. D. Lukin, Phys. Rev. Lett. 84, 5094 (2000).

[12] O. Katz and O. Firstenberg, Nat. Commun. 9, 2074 (2018).

[13] G. Heinze, C. Hubrich, and T. Halfmann, Phys. Rev. Lett. 111, 033601 (2013).

[14] M. Lovrić, D. Suter, A. Ferrier, and P. Goldner, Phys. Rev. Lett. 111, 020503 (2013).

[15] B. S. Ham, Nat. Photonics 3, 518 (2009).

[16] S. Mieth, G. T. Genov, L. P. Yatsenko, N. V. Vitanov, and T. Halfmann, Phys. Rev. A 93, 012312 (2016).

[17] A. Eilam, I. Azuri, A. V. Sharypov, A. D. Wilson-Gordon, and H. Friedmann, Opt. Lett. 35, 772 (2010).

[18] M.-A. Maynard, F. Bretenaker, and F. Goldfarb, Phys. Rev. A 90, 061801(R) (2014).

[19] A. J. F. de Almeida, J. Sales, M.-A. Maynard, T. Lauprêtre, F. Bretenaker, D. Felinto, F. Goldfarb, and J. W. R. Tabosa, Phys. Rev. A 90, 043803 (2014).

[20] A. de Almeida, S. Barreiro, W. Martins, R. de Oliveira, D. Felinto, L. Pruvost, and J. Tabosa, Opt. Lett. 40, 2545 (2015).
[21] R. W. Boyd, M. G. Raymer, P. Narum, and D. J. Harter, Phys. Rev. A 24, 411 (1981).

[22] J. H. Lee, J. Song, M. Scarparo, and M. D. Levenson, Opt. Lett. 5, 196 (1980).

[23] M. Mrozek, A. M. Wojciechowski, D. S. Rudnicki, J. Zachorowski, P. Kehayias, D. Budker, and W. Gawlik, Phys. Rev. B 94, 035204 (2016).

[24] S.-W. Chang, S.-L. Chuang, P.-C. Ku, C. J. Chang-Hasnain, P. Palinginis, and H. Wang, Phys. Rev. B 70, 235333 (2004).

[25] F. Arrieta-Yañez, S. Melle, O. G. Calderón, M. A. Antón, and F. Carreño, Phys. Rev. A 80, 011804(R) (2009).

[26] T. Lauprêtre, S. Kumar, P. Berger, R. Faoro, R. Ghosh, F. Bretenaker, and F. Goldfarb, Phys. Rev. A 85, 051805(R) (2012).

[27] P. Neveu, M.-A. Maynard, R. Bouchez, J. Lugani, R. Ghosh, F. Bretenaker, F. Goldfarb, and E. Brion, Phys. Rev. Lett. 118, 073605 (2017).

[28] A. Peng, M. Johnsson, W. P. Bowen, P. K. Lam, H.-A. Bachor, and J. J. Hope, Phys. Rev. A 71, 033809 (2005).

[29] F. Goldfarb, J. Ghosh, M. David, J. Ruggiero, T. Chanelière, J.-L. Le Gouët, H. Gilles, R. Ghosh, and F. Bretenaker, Europhys. Lett. 82, 54002 (2008).

[30] P. Neveu, C. Banerjee, J. Lugani, F. Bretenaker, E. Brion, and F. Goldfarb, New J. Phys. 20, 083043 (2018).

[31] C. Cohen-Tannoudji, J. Dupont-Roc, and G. Grynberg, AtomPhoton Interactions: Basic Processes and Applications (WileyVCH, Weinheim, 1998).

[32] P. Lambropoulos and D. Petrosyan, Fundamentals of Quantum Optics and Quantum Information (Springer, Berlin, 2007), Vol. 23.

[33] T. W. Mossberg, R. Kachru, S. R. Hartmann, and A. M. Flusberg, Phys. Rev. A 20, 1976 (1979).

[34] W. Tittel, M. Afzelius, T. Chaneliere, R. L. Cone, S. Kröll, S. A. Moiseev, and M. Sellars, Laser Photonics Rev. 4, 244 (2010).

[35] R. W. Boyd, Z. Shi, and P. Milonni, J. Opt. 12, 104007 (2010)

[36] R. Loudon, The Quantum Theory of Light (Oxford University Press, Oxford, UK, 2000).

[37] E. Figueroa, F. Vewinger, J. Appel, and A. Lvovsky, Opt. Lett. 31, 2625 (2006). 